\title{
Role of Management Strategies in Reducing Mortality From Invasive Fungal Disease in Children With Cancer or Receiving Hemopoietic Stem Cell Transplant
}

\author{
A Single Center 30-year Experience
}

\author{
Elio Castagnola, MD, PhD, * Francesca Bagnasco, PhD, † Loredana Amoroso, MD, + Ilaria Caviglia, PhD, * \\ Silvia Caruso, PhD, † Maura Faraci, MD, \& Michaela Calvillo, MD, 7 Cristina Moroni, MD, * \\ Roberto Bandettini, MD, / Giuliana Cangemi, PhD, / Gian Michele Magnano, MD, ** \\ Piero Buffa, MD, $† \dagger$ Andrea Moscatelli, MD, $+\neq$ and Riccardo Haupt, MD $†$
}

\begin{abstract}
Background: In the last decades, several diagnostic and therapeutic strategies have been implemented for management of invasive fungal diseases (IFD) in patients with cancer or receiving allogeneic hemopoietic stem cell transplant. Few data are available on their impact on mortality in children. Methods: All IFD episodes diagnosed at tertiary care center during a 30-year period between 1983 and 2012 were analyzed for 90-day mortality and risk factors. Diagnoses were coded according to international (European Organization for Research and Treatment of Cancer/Invasive Fungal Infections Cooperative Group and the National Institute of Allergy and Infectious Diseases Mycoses Study Group) criteria. Four treatment eras (1983-1990, 1991-1999, 2000-2005 and 2006-2012) were defined according to availability of diagnostic technologies, new antifungal drugs and use of a diagnostic-driven approach without empiric antifungal therapy.

Results: A total of 198 IFD were diagnosed in 191 patients; $71.2 \%$ were proven/probable infections; $39.9 \%$ were caused by yeasts and $31.3 \%$ by molds. Within 90 days from IFD diagnosis, 58 (30.4\%) patients died for a $28.3 \%$ cumulative probability of death. A multivariable analysis showed that the highest risk of death was associated with alternative donor-hemopoietic stem cell transplant [hazard ratio (HR): 3.96] and mold etiology (HR: 1.34). The risk of death significantly decreased across the treatment eras, with almost a 3-fold reduced risk for patients diagnosed during the 2006-2012 period (HR: 0.24). Also if the variable year of diagnosis was considered as continuous, the hazard of death significantly decreased by $5 \%$ per year (HR: 0.95 ).
\end{abstract}

Conclusions: New management strategies resulted in a better prognosis of IFD in children with cancer or hemopoietic stem cell transplant. A diagnostic-driven approach was not associated with an increase in mortality.

Key Words: invasive fungal disease, cancer, hemopoietic stem cell transplant, children

(Pediatr Infect Dis J 2014;33:233-237)

Accepted for publication August 23, 2013.

From the *Infectious Diseases Unit; †Epidemiology and Biostatistics Unit; tOncology Unit; §Hemopoietic Stem Cell Transplant Unit; $\mathbb{\text { Hematology }}$ Unit; ILaboratory of Analysis; **Service of Radiology; †† Surgery Unit; and †+ Intensive Care Unit, "Istituto Giannina Gaslini”, Genova, Italy.

This work was partially supported by: Italian Foundation for Research on Neuroblastoma; Contributi del "Cinque per mille dell'IRPEF-Finanziamento della Ricerca Sanitaria" (5XMILRIC08 DEL. 136/11); Finanziamento della Ricerca Corrente, Ministero della Salute (contributo per la ricerca intramurale; MSALRC DEL. 169/09) and European Network for Cancer Research in Children and Adolescents (ENCCA; FP7 Project number 261474). The authors have no other funding or conflicts of interest to disclose.

Address for correspondence: Elio Castagnola, MD, Infectious Diseases Unit, G. "Istituto Giannina Gaslini”, Largo G. Gaslini 5, 16147 Genova, Italy. E-mail: eliocastagnola@ospedale-gaslini.ge.it.

Copyright $(2013$ by Lippincott Williams \& Wilkins

ISSN: 0891-3668/14/3303-0233

DOI: $10.1097 /$ INF.0000000000000101 nvasive fungal diseases (IFD) represent severe complications in pediatric cancer patients. Their highest incidence is observed among children receiving treatment for acute leukemia (especially myeloid or relapsing lymphoblastic) or in recipients of allogeneic hemopoietic stem cell transplant (HSCT). ${ }^{1-7}$ The prognosis of IFD is often discouraging, and even if pediatric patients seem to show a lower mortality rate than adults, ${ }^{1}$ the risk of death remains high. ${ }^{1,4-8}$

In the last years, new diagnostic approaches including seriated computed tomography (CT) scan and fungal antigen detection $^{9,10}$ as well as the availability of new antifungal drugs have improved the prognosis of IFD also in children. ${ }^{11-13}$ Empiric antifungal therapy of persistent febrile neutropenia has been a cornerstone in the management of cancer patients for many years. However, recent data from adults showed the feasibility of a clinically driven approach, the so-called preemptive or may be better "diagnostic-driven" therapy, calling for the administration of antifungal therapy only in a persistently febrile neutropenic patient with either a positive radiologic (eg, chest CT scan) or microbiologic result (eg, galactomannan detection in serum or bronchoalveolar lavage, glucan detection in serum, cytologic detection of fungal hypha or positive culture on sputum or bronchoalveolar lavage fluid) suggestive of IFD. ${ }^{14,15}$ With this approach, the use of antifungal treatments was less widespread, but mortality rate was not different from that obtained with the standard strategy calling for administration of antifungal therapy in all patients at risk with persistent febrile neutropenia.

In spite of the knowledge that in children serial CT scans and fungal antigen detection, especially galactomannan, may have performances similar to that observed in adults, ${ }^{9,16-20}$ no data are available about the effectiveness of a diagnostic-driven approach for the treatment of IFD in pediatrics, especially in terms of impact on mortality. The most recent guidelines for therapy of febrile neutropenia in children state that "a preemptive approach may be feasible in centers with adequate experience and facilities, [omissis] research describing the safety and effectiveness of this approach is needed". ${ }^{21}$ Aim of the present study was to describe changes in mortality due to IFD in a tertiary care cancer center according to changes in management strategies.

\section{METHODS}

All children with cancer or a non-neoplastic disease requiring allogeneic HSCT admitted at "Istituto Giannina Gaslini," Genoa, Italy (a tertiary care hospital for the treatment of children with cancer and HSCT) between 1983 and 2012 and who developed at least 1 IFD were eligible for this study. 
Institutional database, active since 1970, prospectively collects demographic and baseline clinical information on all children admitted to the department of Hematology and Oncology of Istituto Giannina Gaslini. Data on etiology and localization of all IFD diagnosed since 1983 are also stored in an ad hoc database. Information has been collected retrospectively for cases diagnosed before 1989 and prospectively thereafter. Each infectious case was defined according to the last revision of the criteria adopted by the European Organization for Research and Treatment of Cancer/Invasive Fungal Infections Cooperative Group and the National Institute of Allergy and Infectious Diseases Mycoses Study Group. ${ }^{22}$ All cases occurring before the publication of the criteria were reviewed and retrospectively classified. Some of these IFD have been previously published in studies on epidemiology or therapy of IFD or as case report(s). A list of these publications is available as Supplemental Digital Content 1, http://links.lww.com/INF/B718.

For each episode of IFD the following data were collected on the affected patient: (1) gender; (2) underlying disease [categorized as acute leukemia/lymphoma (including acute lymphoblastic leukemia, non-Hodgkin lymphoma, acute myeloid leukemia, hemophagocytic lymphohistiocytosis or myelodisplastic syndrome), solid tumor, or non-neoplastic disease]; (3) age at diagnosis of IFD; (4) type of treatment for the underlying disease [chemotherapy (for acute leukemia/lymphoma this included both induction and consolidation, and excluded maintenance treatment), autologous HSCT, allogeneic HSCT from a matched related donor or an alternative donor (AD)] ; (5) underlying disease status at infection [relapsing/resistant malignancy or acute graft vs. host disease (aGvHD)]; (6) type of IFD (possible IFD, proven-probable IFD with deep organ involvement, proven isolated fungemia); (7) type of identified pathogen (yeast, mold or none in case of possible IFD) based on histology, culture growth or antigen detection and (8) year of diagnosis of IFD, as surrogate of different management strategies adopted over the years according with the availability of new technologies and drugs (definitions are available as Supplemental Digital Content 2, http://links.lww.com/INF/B716). ${ }^{9}, 16-18,20,23-25$

Mortality was evaluated either at 90 days since IFD diagnosis, it represent a standard for efficacy evaluation of IFD management as in clinical trials, ${ }^{26}$ and at last follow up. Since sometime, it was very difficult to differentiate between death with or because of IFD, we decided to evaluate overall and not attributable mortality. Dataset was closed at December 31, 2012.

\section{Statistical Analysis}

Descriptive statistics were performed in terms of absolute frequencies and percentages for qualitative data, and the Pearson's $\chi^{2}$ test or Fisher's exact test, if appropriate, was applied to compare proportions. Quantitative data were described in terms of median values and $\mathrm{min} / \mathrm{max}$ values due to their non-normal (Gaussian) distribution. In case that the same subject was affected by $>1$ IFD, these were considered as multiple events and risk factors relative to the fungal disease were modeled as a time-dependent covariate by the counting process approach.

The 90-day cumulative risk of death after invasive fungal disease was calculated by the Kaplan-Meier method and the $95 \%$ confidence interval (CI) of the estimates were calculated according to Greenwood's formula. Log-rank test was used to assess differences between risk factors.

In the multivariable analysis, all variables, except "HSCT (yes or no)" that was already incorporated in the "type of treatment of the underlying disease" and "diagnosis of IFD (proven/ probable or possible)" that was strictly associated with the variable "type of identified pathogen (yeast, mold or none for possible IFD)," were included in an extensions of the Cox model with the counting process approach (the Andersen-Gill model). ${ }^{27,28}$ To select the best fit of the model, a backward selection procedure was used. The hazard ratio (HR) with the $95 \%$ CI was evaluated using a robust estimate of variance to incorporate the intraindividual correlation and the likelihood ratio test was calculated to measure the effect of each predictor.

All tests were 2 -tailed and a $P$-value $<0.05$ was considered statistically significant. All analyses were performed using Stata (Stata Corporation, 2009; StataCorp. Stata Statistical Software, Release 11.0 College Station, TX).

\section{RESULTS}

During the 30-year period covered by this study, 198 episodes of IFD were observed in 191 patients. Seven patients had 2 episodes of IFD for a total of 198 infections. Table 1, first column, reports on the demographic characteristics of the patients, their underlying disease and treatment. Male gender was prevalent (60.2\% of cases) and acute leukemia/lymphoma was the most frequent tumor type category (68.1\%). At diagnosis of IFD, many children were $<10$-year old, the infection mostly occurred during standard antineoplastic chemotherapy (59.6\% of cases), or AD-HSCT ( $22.2 \%$ of cases), and was observed in absence of relapsing/refractory underlying disease $(68.2 \%)$. The most frequent diagnosis was proven/probable IFD (71.2\%), and yeasts represented the most frequently identified pathogens (39.9\%). Finally, there was an increase in the mean number of IFD diagnosed per year from the earliest to the last period.

Details on etiology and localization of the 198 IFD are available as Supplemental Digital Content 3, http://links.lww.com/INF/ B717. Candida non-albicans represented the most frequently isolated yeast ( $50 \%$ of cases), while Aspergillus (including cases diagnosed by means of positive galactomannan antigen) represented the most frequently identified mold (74\% of mold infections). As regards localization of IFD with deep organ involvement, lungs were the most frequent localization in proven-probable IFD $(61 \%$, cases), while $9 \%$ of episodes were defined as disseminated.

\section{Analysis of Mortality and Associated Risk Factors}

Within 90 days from diagnosis of IFD, 58 (30.4\%) patients died for a $28.3 \%(95 \%$ CI: $22.5-35.2)$ cumulative probability of death. As shown in Table 1, the factors associated with a significant higher risk of mortality were: infection following HSCT, in particular AD-HSCT, a diagnosis of proven/probable IFD with deep organ involvement and an infection due to molds. Table 2 summarizes the results of the multivariable analysis 90-day mortality. According to this model, children receiving AD-HSCT had an almost 4-fold increased probability of death compared with those receiving standard chemotherapy, while no significant difference was present for children receiving autologous or matched related donor-HSCT as shown by the $95 \%$ CI including 1 . Patients with IFD due to yeasts had reduced risk (HR: 0.38) of death when compared with those with possible IFD, while the risk of death was similar for proven/probable IFD due to molds and possible IFD. In a model in which possible IFD where not considered and yeasts infections where used as reference, children with infection due to molds had a 2.91-fold increased risk of death (95\% CI: 1.41-6.03). A significant decrease in mortality over the years was evident both when time was treated as a categorical variable (delimitated by the availability of new drugs and diagnostic techniques), with a HR of 0.24 in 2006-2012 compared with 1983-1990 (Table 2), or a continuous one, with a $5 \%$ decrease per year (HR: $0.95,95 \%$ CI: $0.91-0.99, P=0.02$ ).

Finally, in a further analysis performed with a purely explorative aim, very long-term survival of patients with at least 1 IFD 
TABLE 1. Analysis of Factors Associated With Mortality Within 90 Days From Diagnosis of IFD in Children Receiving Antineoplastic Chemotherapy or Hemopoietic Stem Cell Transplant

\begin{tabular}{|c|c|c|c|c|}
\hline & Total N (\%) & No of deaths & $\begin{array}{c}\text { Cumulative 90-day probability } \\
\text { of death ( } 95 \% \text { CI) }\end{array}$ & $\begin{array}{c}\text { Log-rank Test, } \\
\text { P-value }\end{array}$ \\
\hline \multicolumn{5}{|l|}{ Gender } \\
\hline Female & $76(39.8)$ & 20 & $25.0(16.7-36.3)$ & 0.5067 \\
\hline \multicolumn{5}{|l|}{ Underlying disease } \\
\hline Leukemia/lymphoma & $130(68.1)$ & 42 & $30.0(22.9-38.7)$ & \multirow[t]{3}{*}{0.1861} \\
\hline Solid tumor & $44(23.0)$ & 9 & $18.2(9.5-33.1)$ & \\
\hline Non-neoplastic disease & $17(8.9)$ & 7 & $41.2(22.2-67.5)$ & \\
\hline \multicolumn{5}{|l|}{ Age at diagnosis of IFD (years) } \\
\hline$<10$ & $114(57.6)$ & 27 & $23.1(16.3-31.9)$ & \multirow[t]{2}{*}{0.0521} \\
\hline$\geq 10$ & $84(42.4)$ & 31 & $35.9(26.4-47.6)$ & \\
\hline \multicolumn{5}{|l|}{ Type of treatment for underlying disease } \\
\hline Chemotherapy & $118(59.6)$ & 25 & $19.8(13.6-28.3)$ & \multirow[t]{3}{*}{0.0002} \\
\hline Autologous HSCT & $22(11.1)$ & 6 & $25.0(11.2-50.1)$ & \\
\hline MRD HSCT & $14(7.1)$ & 4 & $30.8(12.8-62.7)$ & \\
\hline No & $135(68.2)$ & 34 & $24.8(18.3-33.1)$ & \multirow{2}{*}{0.1139} \\
\hline Yes & $63(31.8)$ & 24 & $36.2(25.3-49.9)$ & \\
\hline \multicolumn{5}{|l|}{ Diagnosis of IFD } \\
\hline Invasive possible & $57(28.8)$ & 17 & $29.1(18.9-43.1)$ & \multirow[t]{3}{*}{0.0268} \\
\hline Proven/probable with deep organ involvement & $79(39.9)$ & 29 & $36.0(26.3-47.9)$ & \\
\hline Proven isolated fungemia & $62(31.3)$ & 12 & $18.1(10.4-30.2)$ & \\
\hline \multicolumn{5}{|c|}{ Type of identified pathogen (by means of histology, cultures, antigen detection) } \\
\hline Not identified (possible IFD) & $57(28.8)$ & 17 & $29.1(18.9-43.1)$ & \multirow[t]{3}{*}{0.0008} \\
\hline Yeast & $79(39.9)$ & 14 & $16.7(10.1-26.9)$ & \\
\hline Mold & $62(31.3)$ & 27 & $43.1(31.6-56.8)$ & \\
\hline \multicolumn{5}{|l|}{ Year of diagnosis of IFD, $\mathrm{n}$ (mean/year) } \\
\hline $1983-1990$ & $39(4.9)$ & 13 & $32.4(19.9-49.9)$ & \multirow[t]{3}{*}{0.839} \\
\hline 1991-1999 & $45(5.0)$ & 13 & $27.9(16.9-43.9)$ & \\
\hline $2000-2005$ & $54(9.0)$ & 17 & $28.8(18.5-43.2)$ & \\
\hline
\end{tabular}

$\mathrm{MRD}$, matched related donor; $\mathrm{AD}$, alternative donor.

TABLE 2. Multivariable Analysis of Risk Factors for 90-days Mortality From Invasive Fungal Disease

\begin{tabular}{lccc}
\hline \hline & HR & $95 \%$ CI & LRT, P-value \\
\hline $\begin{array}{c}\text { Type of treatment } \\
\text { for underlying } \\
\text { disease }\end{array}$ & & & \\
Chemotherapy & ref. & - & 0.0005 \\
Autologous & 0.94 & $0.37-2.39$ & \\
HSCT & & & \\
MRD HSCT & 1.49 & $0.54-4.11$ & \\
AD-HSCT & 3.96 & $1.99-7.85$ & \\
Type of identified pathogen (by means of & \\
histology, cultures, antigen detection) & \\
Not identified & ref. & - & \\
(possible IFD) & & & \\
Yeast & 0.38 & $0.16-0.91$ & \\
Mold & 1.34 & $0.68-2.63$ & \\
Year of diagnosis of IFD & & & \\
1983-1990 & ref. & - & \\
1991-1999 & 0.36 & $0.15-0.85$ & \\
2000-2005 & 0.33 & $0.13-0.83$ & \\
2006-2012 & 0.24 & $0.09-0.60$ & \\
\end{tabular}

LRT, likelihood ratio test; ref, reference; MRD, matched related donor; $\mathrm{AD}$, alternative donor. was also evaluated and 52 further patients were found to be dead after a median of 9.4 months (range 3.1-139.2) from diagnosis. The estimated overall 20-year survival after IFD was $35.9 \%$ (95\% CI: 27.5-43.9). Variables significantly associated with poorer survival were AD-HSCT as part of treatment of the underlying disease, earlier era of treatment and relapsing/refractory underlying diseases with a HR of 2.64 (95\% CI: 1.73-4.01; data not shown).

\section{DISCUSSION}

In this study, we analyzed the risk factors associated to 90-day mortality after an IFD diagnosis in children underlying antineoplastic chemotherapy or HSCT. The study period was very long, 30 years, and during this time many changes in management strategies occurred. In spite of the partially retrospective (from 1983 to 1988) data collection and the definition of some episodes due to changes in medical knowledge (European Organization for Research and Treatment of Cancer/Invasive Fungal Infections Cooperative Group and the National Institute of Allergy and Infectious Diseases Mycoses Study Group definitions first time available in 2002, then revisited in 2008) ${ }^{22,29}$ important considerations may be driven from our observations.

The first and, in our opinion, most important observation regards the decrease in mortality observed over the study period. 
As shown by the multivariable analysis, a significant decrease in mortality was documented both when considering the different eras delimited by the availability of new drugs and diagnostic technologies as when the time variable was considered as continuous. This observation is confirmed by other studies, ${ }^{30-33}$ in which reduced mortality in patients with invasive aspergillosis has been reported and mainly attributed to early diagnosis and availability of new antifungal drugs. Noteworthy, the significant reduction of mortality observed in our study was paralleled by an increase of the number of IFD diagnoses. Different factors interact to this observation: on 1 side more aggressive protocols for the treatment of the underlying disease have been introduced into the clinical practice, on the other hand the management strategies contributed to earlier and more accurate diagnoses and there was an increased availability of effective weapons against fungi. However, we must also recognize that other factors not included in the present analysis (eg, different supportive care measures) might impact on improved prognosis of IFD in these patients' populations. In the last period covered by this study, empirical antifungal therapy strategy was nearly completely abandoned in our center, except for rapidly worsening patients pending results of diagnostic investigations. In spite of this "reduction" of treatment and the increase in the number of IFD diagnosed, we observed a significant reduction in mortality. The logical interpretation of these results is that the new diagnostic tools (mainly galactomannan antigen detection and CT scan) consent an early diagnosis of IFD. As a consequence physicians are aware timely of what's going on in their patients and can act accordingly in a thoughtful and not empirical way, thanks also to the availability of effective antifungal drugs with different toxicity profiles, also indirectly suggesting the feasibility of a diagnostic-driven approach for IFD in children.

We have also shown that mortality is also influenced by the etiology of IFD. As expected, patients with yeast infections, mainly represented by isolated Candida fungemia (57 candidemias/79 Candida infections, 72\%) had a better prognosis, ${ }^{1}$ that those with mold infection. A quite puzzling result of multivariable analysis was that children with possible IFD had a worse prognosis than those diagnosed with yeast infection. We have no clear explanation for this phenomenon. A possible interpretation is that a not negligible proportion of possible IFD is represented either by deep organ candidiasis,${ }^{34}$ or true invasive aspergillosis, ${ }^{35}$ that eventually have a worse prognosis compared with isolated fungemias. On the other hand, it is also possible that the "possible" IFD were due to noninfectious causes (eg, pulmonary aGvHD) that have a bad prognosis as well.

IFD following AD-HSCT had the highest mortality. This aspect has been already described in adults ${ }^{31}$ and has been associated with the presence of uncontrolled aGvHD. ${ }^{36}$ Other authors ${ }^{30,37}$ have also described that cancer remission is favorably associated to a better survival after IFD. We could not confirm this observation because in our study the status of underlying disease did not affect the 90-day mortality and was removed from the multivariable analysis. However, when survival analysis of our patients with IFD was extended to the last visit, the presence of uncontrolled underlying disease (resistant/relapsing malignancy or aGvHD) represented a significant risk factor for long-term mortality. In fact while the overall 20 -year survival of children with IFD was $35.9 \%$, the overall survival of children with cancer treated at our center with at least 1 chemotherapy course or receiving HSCT was $73.6 \%$ (95\% CI: $70.8-76.2$; R.H. personal observations). We can speculate that the development of an IFD and its treatment might interfere in some ways, for example, in reducing dose intensity of chemotherapy. An effect of IFD on long-term mortality has also been recently suggested in adults with acute leukemia or receiving allogeneic
HSCT. ${ }^{38,39}$ Further studies are needed to better clarify these possible interferences between IFD and cancer prognosis.

\section{REFERENCES}

1. Castagnola E, Cesaro S, Giacchino M, et al. Fungal infections in children with cancer: a prospective, multicenter surveillance study. Pediatr Infect Dis J. 2006;25:634-639.

2. Castagnola E, Rossi MR, Cesaro S, et al. Incidence of bacteremias and invasive mycoses in children with acute non-lymphoblastic leukemia: results from a multi-center Italian study. Pediatr Blood Cancer. 2010;55:1103-1107.

3. Hale KA, Shaw PJ, Dalla-Pozza L, et al. Epidemiology of paediatric invasive fungal infections and a case-control study of risk factors in acute leukaemia or post stem cell transplant. Br J Haematol. 2010;149:263-272.

4. Lehrnbecher T, Frank C, Engels K, et al. Trends in the postmortem epidemiology of invasive fungal infections at a university hospital. $J$ Infect. 2010;61:259-265.

5. Steinbach WJ, Marr KA, Anaissie EJ, et al. Clinical epidemiology of 960 patients with invasive aspergillosis from the PATH Alliance registry. $J$ Infect. 2012;65:453-464.

6. Castagnola E, Bagnasco F, Faraci M, et al. Incidence of bacteremias and invasive mycoses in children undergoing allogeneic hematopoietic stem cell transplantation: a single center experience. Bone Marrow Transplant. 2008;41:339-347.

7. Sung L. Invasive fungal infections in children with cancer. $J$ Pediatr. 2010;156:S68-S73.

8. Dvorak CC, Steinbach WJ, Brown JM, et al. Risks and outcomes of invasive fungal infections in pediatric patients undergoing allogeneic hematopoietic cell transplantation. Bone Marrow Transplant. 2005;36:621-629.

9. Archibald S, Park J, Geyer JR, et al. Computed tomography in the evaluation of febrile neutropenic pediatric oncology patients. Pediatr Infect Dis J. 2001;20:5-10.

10. Roilides E, Pana ZD. Application of diagnostic markers to invasive aspergillosis in children. Ann N Y Acad Sci. 2012;1272:1-8.

11. Walsh TJ, Lutsar I, Driscoll T, et al. Voriconazole in the treatment of aspergillosis, scedosporiosis and other invasive fungal infections in children. Pediatr Infect Dis J. 2002;21:240-248.

12. Zaoutis TE, Jafri HS, Huang LM, et al. A prospective, multicenter study of caspofungin for the treatment of documented Candida or Aspergillus infections in pediatric patients. Pediatrics. 2009;123:877-884.

13. Kolve H, Ahlke E, Fegeler W, et al. Safety, tolerance and outcome of treatment with liposomal amphotericin B in paediatric patients with cancer or undergoing haematopoietic stem cell transplantation. J Antimicrob Chemother. 2009;64:383-387.

14. Girmenia C, Micozzi A, Gentile G, et al. Clinically driven diagnostic antifungal approach in neutropenic patients: a prospective feasibility study. J Clin Oncol. 2010;28:667-674.

15. Cordonnier $\mathrm{C}$, Pautas $\mathrm{C}$, Maury $\mathrm{S}$, et al. Empirical versus preemptive antifungal therapy for high-risk, febrile, neutropenic patients: a randomized, controlled trial. Clin Infect Dis. 2009;48:1042-1051.

16. Taccone A, Occhi M, Garaventa A, et al. CT of invasive pulmonary aspergillosis in children with cancer. Pediatr Radiol. 1993;23:177-180.

17. Burgos A, Zaoutis TE, Dvorak CC, et al. Pediatric invasive aspergillosis: a multicenter retrospective analysis of 139 contemporary cases. Pediatrics. 2008;121:e1286-e1294

18. Castagnola E, Furfaro E, Caviglia I, et al. Performance of the galactomannan antigen detection test in the diagnosis of invasive aspergillosis in children with cancer or undergoing haemopoietic stem cell transplantation. Clin Microbiol Infect. 2010;16:1197-1203.

19. Mularoni A, Furfaro E, Faraci M, et al. High Levels of beta-D-glucan in immunocompromised children with proven invasive fungal disease. Clin Vaccine Immunol. 2010;17:882-883.

20. Steinbach WJ, Addison RM, McLaughlin L, et al. Prospective Aspergillus galactomannan antigen testing in pediatric hematopoietic stem cell transplant recipients. Pediatr Infect Dis J. 2007;26:558-564.

21. Lehrnbecher T, Phillips R, Alexander S, et al.; International Pediatric Fever and Neutropenia Guideline Panel. Guideline for the management of fever and neutropenia in children with cancer and/or undergoing hematopoietic stem-cell transplantation. J Clin Oncol. 2012;30:4427-4438.

22. De Pauw B, Walsh TJ, Donnelly JP, et al.; European Organization for Research and Treatment of Cancer/Invasive Fungal Infections Cooperative Group; National Institute of Allergy and Infectious Diseases Mycoses Study Group (EORTC/MSG) Consensus Group. Revised definitions of invasive 
fungal disease from the European Organization for Research and Treatment of Cancer/Invasive Fungal Infections Cooperative Group and the National Institute of Allergy and Infectious Diseases Mycoses Study Group (EORTC/ MSG) Consensus Group. Clin Infect Dis. 2008;46:1813-1821 .

23. Walsh TJ, Karlsson MO, Driscoll T, et al. Pharmacokinetics and safety of intravenous voriconazole in children after single- or multiple-dose administration. Antimicrob Agents Chemother. 2004;48:2166-2172.

24. Pasqualotto AC, Shah M, Wynn R, et al. Voriconazole plasma monitoring. Arch Dis Child. 2008;93:578-581.

25. Walsh TJ, Adamson PC, Seibel NL, et al. Pharmacokinetics, safety, and tolerability of caspofungin in children and adolescents. Antimicrob Agents Chemother. 2005;49:4536-4545.

26. Segal BH, Herbrecht R, Stevens DA, et al. Defining responses to therapy and study outcomes in clinical trials of invasive fungal diseases: Mycoses Study Group and European Organization for Research and Treatment of Cancer consensus criteria. Clin Infect Dis. 2008;47:674-683.

27. Kelly PJ, Lim LL. Survival analysis for recurrent event data: an application to childhood infectious diseases. Stat Med. 2000;19:13-33.

28. Therneau TM, Grambsch PM. Modeling Survival Data: Extending the Cox Model. New York: Springer; 2000.

29. Ascioglu S, Rex JH, de Pauw B, et al.; Invasive Fungal Infections Cooperative Group of the European Organization for Research and Treatment of Cancer; Mycoses Study Group of the National Institute of Allergy and Infectious Diseases. Defining opportunistic invasive fungal infections in immunocompromised patients with cancer and hematopoietic stem cell transplants: an international consensus. Clin Infect Dis. 2002;34:7-14.

30. Hahn-Ast C, Glasmacher A, Mückter S, et al. Overall survival and fungal infection-related mortality in patients with invasive fungal infection and neutropenia after myelosuppressive chemotherapy in a tertiary care centre from 1995 to 2006. JAntimicrob Chemother. 2010;65:761-768.

31. Parody R, Martino R, Sánchez F, et al. Predicting survival in adults with invasive aspergillosis during therapy for hematological malignancies or after hematopoietic stem cell transplantation: Single-center analysis and validation of the Seattle, French, and Strasbourg prognostic indexes. Am J Hematol. 2009;84:571-578.

32. Upton A, Kirby KA, Carpenter P, et al. Invasive aspergillosis following hematopoietic cell transplantation: outcomes and prognostic factors associated with mortality. Clin Infect Dis. 2007;44:531-540.

33. Caillot $\mathrm{D}$, Latrabe V, Thiébaut A, et al. Computer tomography in pulmonary invasive aspergillosis in hematological patients with neutropenia: an useful tool for diagnosis and assessment of outcome in clinical trials. Eur J Radiol. 2010;74:e172-e175.

34. Horn DL, Ostrosky-Zeichner L, Morris MI, et al. Factors related to survival and treatment success in invasive candidiasis or candidemia: a pooled analysis of two large, prospective, micafungin trials. Eur J Clin Microbiol Infect Dis. 2010;29:223-229.

35. Subirà $\mathrm{M}$, Martino R, Rovira $\mathrm{M}$, et al. Clinical applicability of the new EORTC/MSG classification for invasive pulmonary aspergillosis in patients with hematological malignancies and autopsy-confirmed invasive aspergillosis. Ann Hematol. 2003;82:80-82.

36. Cordonnier C, Ribaud P, Herbrecht R, et al.; Société Française de Greffe de Moelle et de Thérapie Cellulaire. Prognostic factors for death due to invasive aspergillosis after hematopoietic stem cell transplantation: a 1-year retrospective study of consecutive patients at French transplantation centers. Clin Infect Dis. 2006;42:955-963.

37. Ribrag V, Dreyfus F, Venot A, et al. Prognostic factors of invasive pulmonary aspergillosis in leukemic patients. Leuk Lymphoma. 1993;10:317-321.

38. Even C, Bastuji-Garin S, Hicheri Y, et al. Impact of invasive fungal disease on the chemotherapy schedule and event-free survival in acute leukemia patients who survived fungal disease: a case-control study. Haematologica. 2011;96:337-341.

39. Salmeron G, Porcher R, Bergeron A, et al. Persistent poor long-term prognosis of allogeneic hematopoietic stem cell transplant recipients surviving invasive aspergillosis. Haematologica. 2012;97:1357-1363. 